\title{
BOUNDARY BEHAVIOR OF THE BERGMAN KERNEL FUNCTION ON SOME PSEUDOCONVEX DOMAINS IN $\mathbf{C}^{n}$
}

\author{
SANGHYUN CHO
}

\begin{abstract}
Let $\Omega$ be a bounded pseudoconvex domain in $\mathbf{C}^{n}$ with smooth defining function $r$ and let $z_{0} \in b \Omega$ be a point of finite type. We also assume that the Levi form $\partial \bar{\partial} r(z)$ of $b \Omega$ has $(n-2)$-positive eigenvalues at $z_{0}$. Then we get a quantity which bounds from above and below the Bergman kernel function in a small constant and large constant sense.
\end{abstract}

\section{INTRODUCTION}

Let $\Omega \subset \mathbb{C}^{n}$ be a bounded domain in $\mathbb{C}^{n}$. A natural operator on $\Omega$ is the orthogonal projection

$$
P: L^{2}(\Omega) \longrightarrow H(\Omega) \cap L^{2}(\Omega)=A^{2}(\Omega)
$$

where $H(\Omega)$ denotes the holomorphic functions on $\Omega$. There is a corresponding kernel function $K(z, \bar{z})$, the Bergman kernel function, given by

$$
K_{\Omega}(z, \bar{z})=\sup \left\{|f(z)|^{2} ; f \in A^{2}(\Omega),\|f\|_{L^{2}(\Omega)} \leq 1\right\} .
$$

Since the important paper of Fefferman [11], the singularity of the Bergman kernel function and the asymptotic behavior of the Bergman metric on strongly pseudoconvex domains at the boundary are quite well known. For weakly pseudoconvex domains, however, much less is known. Herbort [12] obtained estimates of the Bergman kernel function for the pseudoconvex domains of homogeneous finite diagonal type in $\mathbb{C}^{n}$ (see section 1 of [12] for the definition). Also McNeal [15] obtained lower bounds on the Bergman metric near a point where a subelliptic estimate of order $\epsilon$ holds on $(0,1)$-forms for the $\bar{\partial}$-Neumann problem. He used lower bounds of the Bergman kernel function near a point of finite type. Estimates also have been obtained for some weakly pseudoconvex domains in $\mathbb{C}^{n}$, but in each case the lower bounds are different from the upper bounds $[1,8,9,10,16]$. In [3], Catlin got a result which completely characterized the boundary behavior of $K_{\Omega}(z, \bar{z})$ for weakly pseudoconvex domains of finite type in $\mathbb{C}^{2}$. Let $\Omega$ be a smoothly bounded pseudoconvex domain in $\mathbb{C}^{n}$ with smooth defining function $r$ and let $z_{0} \in b \Omega$ be a point of finite type $m$ in the sense of D'Angelo [7]. The purpose of this paper is to characterize the boundary behavior of $K(z, \bar{z})$ for $z$ near a point $z_{0} \in b \Omega$ where

Received by the editors April 27, 1993 and, in revised form, December 20, 1993.

1991 Mathematics Subject Classification. Primary 32H15.

Key words and phrases. Bergman kernel function, finite 1-type, plurisubharmonic functions.

Partially supported by Nondirected research fund, K. R. F. 1993, and by GARC-KOSEF. 
the Levi form of $b \Omega$ has $(n-2)$-positive eigenvalues. Note that the type $m$ at $z_{0}$ is an even integer in this case. A particular case has been handled by D'Angelo [6]. He got an exact formula of $K(z, \bar{z})$ for the domains defined by $r(z)=\left|z_{n}\right|^{2 m}+\sum_{i=1}^{n-1}\left|z_{i}\right|^{2}-1$.

We assume that $\frac{\partial r}{\partial z_{1}}(z) \neq 0$ for all $z$ in a neighborhood $U$ of $z_{0}$. After a linear change of coordinates, we can find coordinate functions $z_{1}, \ldots, z_{n}$ defined on $U$ such that

$$
\begin{array}{r}
L_{1}=\frac{\partial}{\partial z_{1}}, \quad L_{j}=\frac{\partial}{\partial z_{j}}+b_{j} \frac{\partial}{\partial z_{1}}, \quad L_{j} r \equiv 0, \quad b_{j}\left(z_{0}\right)=0, \\
j=2, \ldots, n,
\end{array}
$$

which form a basis of $\mathbb{C} T^{(1,0)}(U)$ and satisfy

$$
\partial \bar{\partial} r\left(z_{0}\right)\left(L_{i}, \bar{L}_{j}\right)=\delta_{i j}, \quad 2 \leq i, j \leq n-1,
$$

where $\delta_{i j}=1$ if $i=j$ and $\delta_{i j}=0$ otherwise. For any integers $j, k>0$, set

$$
\mathscr{L}_{j, k} \partial \bar{\partial} r(z)=\underbrace{L_{n} \ldots L_{n}}_{(j-1) \text { times }} \underbrace{\bar{L}_{n} \ldots \bar{L}_{n}}_{(k-1) \text { times }} \partial \bar{\partial} r(z)\left(L_{n}, \bar{L}_{n}\right),
$$

and define

$$
C_{l}(z)=\max \left\{\left|\mathscr{L}_{j, k} \partial \bar{\partial} r(z)\right| ; j+k=l\right\} .
$$

We can state the main result as follows.

Theorem 1. Let $\Omega$ be a smoothly bounded pseudoconvex domain in $\mathbf{C}^{n}$ and let $z_{0}$ be a point of finite type $m$ on $b \Omega$. Also assume that the Levi-form of $b \Omega$ has $(n-2)$-positive eigenvalues at $z_{0}$. Then there exist a neighborhood $U$ of $z_{0}$ and $a$ constant $C$ such that

$$
\frac{1}{C} \sum_{l=2}^{m} C_{l}(z)^{i}|r(z)|^{-n-\xi} \leq K_{\Omega}(z, \bar{z}) \leq C \sum_{l=2}^{m} C_{l}(z)^{i}|r(z)|^{-n-\bar{q}}
$$

for all $z \in U$, where $C_{l}(z)$ is defined as in (1.3).

Remark 1.1. Since $z_{0} \in b \Omega$ is a point of finite type $m$, we have $C_{m}\left(z_{0}\right)>0$. Therefore (1.4) says, in particular, that

$$
K_{\Omega}(z, \bar{z}) \geq c^{\prime}|r(z)|^{-n-\frac{2}{m}}
$$

for all $z \in U$, for some $c^{\prime}>0$.

Remark 1.2. For pseudoconvex domains $\Omega \subset \subset \mathbb{C}^{n}$ with real analytic smooth boundaries, Kohn [14] conjectured that $K_{\Omega}(z, \bar{z}) \geq c|r(z)|^{-k-2-\epsilon}$ where $\epsilon>$ 0 , and $k$ is the number of positive eigenvalues of the Levi form of $b \Omega$ at $z_{0} \in b \Omega$. In [10], Diederich, Herbort, and Ohsawa proved that the Bergman kernel function satisfies

$$
K_{\Omega}(z, \bar{z}) \geq c|r(z)|^{-k-2-\epsilon_{k}}\left[\log \left(\frac{1}{r(z)}\right)\right]^{-1}
$$

near $z_{0} \in b \Omega$ if the Levi-form of $b \Omega$ at $z_{0}$ has $k$ positive eigenvalues and $\Omega$ is uniformly extendable in a pseudoconvex way of order $N$ (This means that there are neighborhoods $U, V$ of $z_{0} \in b \Omega, V \subset \subset U$, so that for each 
$z^{\prime} \in V \cap b \Omega$, there is a pseudoconvex hypersurface that meets $b \Omega$ only at $z^{\prime}$ and escapes from $b \Omega$ at least of polynomial order $N$ in $U$. See [10, Definition 1] for a detailed definition). Here $\epsilon_{k}=2(n-k-1) / N$ if $k \leq n-2$ and $\epsilon_{n-1}=0$. If $z_{0} \in b \Omega$ is a point of finite type, then $N \geq m$. In [8], Diederich and Fornaess proved that a pseudoconvex domain with real analytic boundary is uniformly extendable of some order. Recently, the author showed the same result in case $b \Omega$ is pseudoconvex and finite type [5]. The main theorem completely characterizes the boundary behavior of $K_{\Omega}(z, \bar{z})$ in $\mathbb{C}^{n}$, in case $z_{0} \in b \Omega$ is of finite type and the Levi form has $(n-2)$-positive eigenvalues, while (1.5) gives a lower bound of $K_{\Omega}(z, \bar{z})$ in this case.

A key idea to prove Theorem 1 is that the terms mixed with strongly pseudoconvex directions and weakly pseudoconvex directions can be negligible. This result will be proved in several propositions in section 2 . Then the proof of Theorem 1 is based on the construction of special polydiscs and weighted $L_{2}$ estimates of Hörmander which Catlin has employed to get a result for $K_{\Omega}(z, \bar{z})$ in $\mathbb{C}^{2}$.

I would like to thank D.W. Catlin and Alan Noell for several conversations we had about the material in this paper.

\section{SPECIAL COORDINATES AND POLYDISCS}

In this section we want to show that about each point $z^{\prime}$ in $U$, there is a polydisc (more precisely, the biholomorphic image of a polydisc) of maximal size on which the function $r(z)$ changes by no more than some prescribed small number $\delta>0$. First we show how to construct the coordinates about $z^{\prime}$ which will be used to define a polydisc.

Let us take the coordinate functions $z_{1}, \ldots, z_{n}$ about $z_{0}$ so that (1.2) holds. Therefore $\left|L_{1} r(z)\right| \geq c>0$ for all $z \in U$, and $\partial \bar{\partial} r(z)\left(L_{i}, \bar{L}_{j}\right)_{2 \leq i, j \leq n-1}$ has $(n-2)$-positive eigenvalues in $U$ where

$$
\begin{aligned}
& L_{1}=\frac{\partial}{\partial z_{1}}, \text { and } \\
& L_{j}=\frac{\partial}{\partial z_{j}}-\left(\frac{\partial r}{\partial z_{1}}\right)^{-1} \frac{\partial r}{\partial z_{j}} \frac{\partial}{\partial z_{1}}, \quad j=2, \ldots, n .
\end{aligned}
$$

Set

$$
\begin{aligned}
& w_{1}=z_{1}+\sum_{j=2}^{n}\left[\left(\frac{\partial r}{\partial z_{1}}\right)^{-1} \frac{\partial r}{\partial z_{j}}\left(z^{\prime}\right)\right] z_{j}, \text { and } \\
& w_{j}=z_{j} \text { for } j=2, \ldots, n .
\end{aligned}
$$

Then $L_{j}$ can be written as

$$
L_{j}=\frac{\partial}{\partial w_{j}}+b_{j}^{\prime} \frac{\partial}{\partial w_{1}}, \quad 2 \leq j \leq n,
$$

where $b_{j}^{\prime}\left(z^{\prime}\right)=0$. In $w_{1}, \ldots, w_{n}$ coordinates, $A=\left(\frac{\partial^{2} r\left(z^{\prime}\right)}{\partial w_{i} \partial \bar{w}_{j}}\right)_{2 \leq i, j \leq n-1}$ is an Hermitian matrix and there is a unitary matrix $P=\left(P_{i j}\right)_{2 \leq i, j \leq n-1}$ such that 
$P^{*} A P=D$, where $D$ is a diagonal matrix whose entries are positive eigenvalues of $A$. Set

$$
\begin{aligned}
& z_{1}=w_{1}, \quad z_{n}=w_{n}, \quad \text { and } \\
& z_{j}=\sum_{k=2}^{n-1} \bar{P}_{k j} w_{k}, \quad \text { for } j=2, \ldots, n-1
\end{aligned}
$$

Then $\frac{\partial^{2} r}{\partial z_{i} \partial z_{j}}\left(z^{\prime}\right)=\lambda_{i} \delta_{i j}, 2 \leq i, j \leq n-1$, where $\lambda_{i}>0$ is an $i$ th entry of $D$ (we may assume that $\lambda_{i} \geq c>0$ in $U$ for all $i$ ). Finally set $w_{j}=\lambda_{j}^{-\frac{1}{2}} z_{j}$, $j=2, \ldots, n-1, w_{1}=z_{1}, w_{n}=z_{n}$. Then

$$
\frac{\partial^{2} r}{\partial w_{i} \partial \bar{w}_{j}}\left(z^{\prime}\right)=\delta_{i j}, \quad 2 \leq i, j \leq n-1 .
$$

Remark 2.1. If we take the above coordinate changes to get (2.2) with $z^{\prime}$ replaced by $z_{0}$, then this coordinate function satisfies (1.2).

Proposition 2.1. For each positive number $\epsilon>0$, there is a neighborhood $U_{\epsilon}$ of $z_{0}$ such that

$$
\left|\partial \bar{\partial} r(z)\left(L_{i}, \bar{L}_{j}\right)\right| \leq \epsilon
$$

for all $z \in U_{\epsilon}$ and $2 \leq i, j \leq n-1, i \neq j$.

Proof. From Remark 2.1, and from the coordinate changes up to (2.2), one has $L_{j}=\sum_{k=2}^{n-1} b_{j k} \frac{\partial}{\partial w_{k}}+b_{j}^{\prime} \frac{\partial}{\partial w_{1}}$, where $b_{j}^{\prime}\left(z_{0}\right)=0$ and $\partial \bar{\partial} r\left(L_{i}, \bar{L}_{j}\right)\left(z_{0}\right)=\delta_{i j}$. So (2.3) holds provided one takes $U_{\epsilon}$ sufficiently small.

Proposition 2.2. For each $z^{\prime} \in U$ and positive even integer $m$, there is a biholomorphism $\Phi_{z^{\prime}}: \mathbb{C}^{n} \longrightarrow \mathbb{C}^{n}, \Phi_{z^{\prime}}^{-1}\left(z^{\prime}\right)=0, \Phi_{z^{\prime}}^{-1}(z)=\left(\zeta_{1}, \ldots, \zeta_{n}\right)$ such that

$$
\begin{aligned}
r\left(\Phi_{z^{\prime}}(\zeta)\right)= & r\left(z^{\prime}\right)+\operatorname{Re} \zeta_{1}+\sum_{\substack { \alpha=2 \\
\begin{subarray}{c}{j-1 \\
j, k>0 m{ \alpha = 2 \\
\begin{subarray} { c } { j - 1 \\
j , k > 0 m } }\end{subarray}} \operatorname{Re}\left(b_{j, k}^{\alpha}\left(z^{\prime}\right) \zeta_{n}^{j} \bar{\zeta}_{n}^{k} \zeta_{\alpha}\right) \\
& +\sum_{\substack{j+k \leq m \\
j, k>0}} a_{j, k}\left(z^{\prime}\right) \zeta_{n}^{j} \bar{\zeta}_{n}^{k}+\sum_{\alpha=2}^{n-1}\left|\zeta_{\alpha}\right|^{2} \\
& +\theta\left(\left|\zeta_{1}\right||\zeta|+\left|\zeta^{\prime \prime}\right|^{2}|\zeta|+\left|\zeta^{\prime \prime}\right|\left|\zeta_{n}\right|^{\frac{m}{2}+1}+\left|\zeta_{n}\right|^{m+1}\right) .
\end{aligned}
$$

Proof. We may assume that $z^{\prime}=0 \in b \Omega$. Let us take the coordinate functions $w_{1}, \ldots, w_{n}$ about 0 so that $(2.2)$ holds. After a linear change, $r(w)$ can be written as

$$
\begin{aligned}
r(w)= & \operatorname{Re} w_{1}+\sum_{\alpha=2}^{n-1} \sum_{1 \leq j \leq \frac{m}{2}} \operatorname{Re}\left[\left(a_{j}^{\alpha} w_{n}^{j}+b_{j}^{\alpha} \bar{w}_{n}^{j}\right) w_{\alpha}\right] \\
& +\sum_{\alpha=2}^{n-1} \sum_{\substack{j+k \leq \frac{m}{2} \\
j, k>0}} \operatorname{Re}\left(a_{j, k}^{\alpha} w_{n}^{j} \bar{w}_{n}^{k} w_{\alpha}\right)+\sum_{2 \leq j+k \leq m} b_{j, k} w_{n}^{j} \bar{w}_{n}^{k}+\sum_{\alpha=2}^{n-1}\left|w_{\alpha}\right|^{2} \\
& +\mathcal{O}\left(\left|w_{1}\left\|\left.w|+| w^{\prime \prime}\right|^{2}|w|+\left|w^{\prime \prime} \| w_{n}\right|^{\frac{m}{2}+1}+\left|w_{n}\right|^{m+1}\right),\right.\right.
\end{aligned}
$$


where $w^{\prime \prime}=\left(0, w_{2}, \ldots, w_{n-1}, 0\right)$. It is standard to perform the change of coordinates

$$
\begin{aligned}
& z_{1}=w_{1}+\sum_{2 \leq k \leq m} \frac{2}{k !} \frac{\partial^{k} r(0)}{\partial w_{n}^{k}} w_{n}^{k}+\sum_{\alpha=2}^{n-1} \sum_{1 \leq k \leq m} \frac{2}{k !} \frac{\partial^{k+1} r(0)}{\partial w_{\alpha} \partial w_{n}^{k}} w_{\alpha} w_{n}^{k}, \\
& z_{j}=w_{j}, \quad j=2, \ldots, n,
\end{aligned}
$$

which serves to remove the pure terms from (2.5), i.e., it removes $w_{n}^{k}, \bar{w}_{n}^{k}$ terms as well as $w_{n}^{k} w_{\alpha}, \bar{w}_{n}^{k} \bar{w}_{\alpha}$ terms from the summation in (2.5). We may also perform a change of coordinates,

$$
\zeta_{1}=z_{1}, \quad \zeta_{n}=z_{n}, \quad \zeta_{\alpha}=z_{\alpha}+\sum_{1 \leq k \leq \frac{\pi}{2}} \frac{1}{k !} \frac{\partial^{k+1} r(0)}{\partial \bar{w}_{\alpha} \partial w_{n}^{k}} z_{n}^{k}
$$

to remove terms of the form $\bar{w}_{n}^{j} w_{\alpha}$ from the summation in (2.5), and hence $r(\zeta)$ has the desired expression as in (2.4) in $\zeta$-coordinates.

Remark 2.2. The coordinate changes in the proof of Proposition 2.2 are unique and hence the map $\Phi_{z^{\prime}}$ is defined uniquely.

Set $\rho(\zeta)=r \circ \Phi_{z^{\prime}}(\zeta)$, and set

$$
\begin{aligned}
& A_{l}\left(z^{\prime}\right)=\max \left\{\left|a_{j, k}\left(z^{\prime}\right)\right| ; j+k=l\right\}, \quad 2 \leq l \leq m, \\
& B_{l^{\prime}}\left(z^{\prime}\right)=\max \left\{\left|b_{j, k}^{\alpha}\left(z^{\prime}\right)\right| ; j+k=l^{\prime}, \quad 2 \leq \alpha \leq n-1\right\}, \quad 2 \leq l^{\prime} \leq \frac{m}{2} .
\end{aligned}
$$

For each $\delta>0$, we define $\tau\left(z^{\prime}, \delta\right)$ as follows:

(2.7) $\tau\left(z^{\prime}, \delta\right)=\min \left\{\left(\delta / A_{l}\left(z^{\prime}\right)\right)^{\dagger},\left(\delta^{\frac{1}{2}} / B_{l^{\prime}}\left(z^{\prime}\right)\right)^{\frac{1}{t}} ; 2 \leq l \leq m, 2 \leq l^{\prime} \leq m / 2\right\}$.

Since $A_{m}\left(z_{0}\right) \geq c>0$, it follows that $A_{m}\left(z^{\prime}\right) \geq c^{\prime}>0$ for all $z^{\prime} \in U$ if $U$ is sufficiently small. This gives the inequality,

$$
\delta^{\frac{1}{2}} \lesssim \tau\left(z^{\prime}, \delta\right) \lesssim \delta^{\frac{1}{m}}, \quad z^{\prime} \in U .
$$

The definition of $\tau\left(z^{\prime}, \delta\right)$ easily implies that if $\delta^{\prime}<\delta^{\prime \prime}$, then

$$
\left(\delta^{\prime} / \delta^{\prime \prime}\right)^{\frac{1}{2}} \tau\left(z^{\prime}, \delta^{\prime \prime}\right) \leq \tau\left(z^{\prime}, \delta^{\prime}\right) \leq\left(\delta^{\prime} / \delta^{\prime \prime}\right)^{\frac{1}{m}} \tau\left(z^{\prime}, \delta^{\prime \prime}\right) .
$$

Now set $\tau_{1}=\delta, \tau_{2}=\ldots=\tau_{n-1}=\delta^{\frac{1}{2}}, \tau_{n}=\tau\left(z^{\prime}, \delta\right)=\tau$ and define

$$
\begin{aligned}
& \boldsymbol{R}_{\delta}\left(z^{\prime}\right)=\left\{\zeta \in \mathbb{C}^{n} ;\left|\zeta_{k}\right|<\tau_{k}, k=1,2, \ldots, n\right\}, \text { and } \\
& Q_{\delta}\left(z^{\prime}\right)=\left\{\Phi_{z^{\prime}}(\zeta) ; \zeta \in R_{\delta}\left(z^{\prime}\right)\right\} .
\end{aligned}
$$

In the sequal we denote $D_{k}^{l}$ any partial derivative operator of the form $\frac{\partial^{\mu+\nu}}{\partial \zeta_{k}^{\mu} \partial \bar{\zeta}_{k}^{\nu}}$, where $\mu+\nu=l, k=1,2, \ldots, n$.

Proposition 2.3. Let $z^{\prime} \in U$. Then the function $\rho=r \circ \Phi_{z^{\prime}}(\zeta)$ satisfies

$$
\begin{aligned}
& |\rho(\zeta)-\rho(0)| \lesssim \delta, \quad \zeta \in R_{\delta}\left(z^{\prime}\right), \text { and } \\
& \left|D_{k}^{i} D_{n}^{l} \rho(\zeta)\right| \lesssim \delta \tau_{n}^{-l} \tau_{k}^{-i}, \quad \zeta \in R_{\delta}\left(z^{\prime}\right),
\end{aligned}
$$

for $l+\frac{i m}{2} \leq m, i=0,1, k=2, \ldots, n-1$.

Proof. The definitions in (2.6) and (2.7) imply that $\left|D_{n}^{j} \rho(0)\right| \lesssim \delta \tau^{-j}$ and $\left|D_{k} D_{n}^{j} \rho(0)\right| \lesssim \delta^{\frac{1}{2}} \tau^{-j}=\delta \tau^{-j} \tau_{k}^{-1}$. Since $\left|D_{k} D_{n}^{\frac{m}{2}+1} \rho\left(0, \ldots, \zeta_{k}, 0, \ldots, \zeta_{n}\right)\right| \lesssim$ 
1 , for $k=2, \ldots, n-1$, and $\left|D_{n}^{m+1} \rho\left(0, \zeta_{n}\right)\right| \lesssim 1$, we may use (2.4) and Taylor's expansion theorem to prove (2.11).

In order to study how $\tau(z, \delta)$ depends on $z$ for $z \in Q_{\delta}\left(z^{\prime}\right)$, it is convenient to introduce an analogous quantity $\eta(z, \delta)$ that is defined more intrinsically. Recall that $L_{n}$ is given by

$$
L_{n}=\frac{\partial}{\partial z_{n}}-\left(\frac{\partial r}{\partial z_{1}}\right)^{-1} \frac{\partial r}{\partial z_{n}} \frac{\partial}{\partial z_{1}}
$$

For any $j, k$ with $j, k>0$, define

$$
\mathscr{L}_{j, k} \partial \bar{\partial} r(z)=\underbrace{L_{n} \ldots L_{n}}_{(j-1) \text { times }} \underbrace{\bar{L}_{n} \ldots \bar{L}_{n}}_{(k-1) \text { times }} \partial \bar{\partial} r\left(L_{n}, \bar{L}_{n}\right)(z),
$$

and define

$$
\begin{array}{ll}
\text { (2.12) } & C_{l}(z)=\max \left\{\left|\mathscr{L}_{j, k} \partial \bar{\partial} r(z)\right| ; j+k=l\right\}, \quad l=2, \ldots, m, \\
\text { (2.13) } & \eta(z, \delta)=\min \left\{\left(\delta / C_{l}(z)\right)^{\dagger} ; l=2, \ldots, m\right\} .
\end{array}
$$

Set $L_{n}^{\prime}=\left(d \Phi_{z^{\prime}}\right)^{-1} L_{n}$, and define

$$
\mathscr{L}_{j, k}^{\prime} \partial \bar{\partial} \rho(\zeta)=\underbrace{L_{n}^{\prime} \ldots L_{n}^{\prime}}_{(j-1) \text { times }} \underbrace{\bar{L}_{n}^{\prime} \ldots \bar{L}_{n}^{\prime}}_{(k-1) \text { times }} \partial \bar{\partial} \rho(\zeta)\left(L_{n}^{\prime}, \bar{L}_{n}^{\prime}\right) \text {. }
$$

Then

$$
\mathscr{L}_{j, k} \partial \bar{\partial} r\left(\Phi_{z^{\prime}}(\zeta)\right)=\mathscr{L}_{j, k}^{\prime} \partial \bar{\partial} \rho(\zeta)
$$

by functoriality. Notice that

$$
\begin{aligned}
\left(\Phi_{z^{\prime}}^{-1}\right)_{*} L_{n} & =L_{n}^{\prime}=\frac{\partial}{\partial \zeta_{n}}+b(\zeta) \frac{\partial}{\partial \zeta_{1}}, \text { and } \\
\left(\Phi_{z^{\prime}}^{-1}\right)_{*} L_{k} & =L_{k}^{\prime}=\sum_{j=2}^{n-1} \bar{P}_{k j} \lambda_{j}^{-\frac{1}{2}} \frac{\partial}{\partial \zeta_{j}}-\left(\frac{\partial r}{\partial \zeta_{1}}\right)^{-1} \sum_{j=2}^{n-1} \bar{P}_{k j} \lambda_{j}^{-\frac{1}{2}} \frac{\partial r}{\partial \zeta_{j}} \frac{\partial}{\partial \zeta_{1}} \\
& =\sum_{j=1}^{n-1} b_{k j} \frac{\partial}{\partial \zeta_{j}}, \quad k=2, \ldots, n-1,
\end{aligned}
$$

where $b(\zeta)=-\left(\frac{\partial \rho}{\partial \zeta_{1}}\right)^{-1}\left(\frac{\partial \rho}{\partial \zeta_{n}}\right)$ and $P=\left(P_{k j}\right)$ is a unitary matrix. Since $\frac{\partial \rho}{\partial \zeta_{1}}(\zeta) \neq 0$ in $\Phi_{z^{\prime}}^{-1}(U)$, we obtain from Leibniz's identity and (2.11) that

$$
\left|D_{k}^{i} D_{n}^{l} b(0)\right| \lesssim \delta \tau^{-l-1} \tau_{k}^{-i},
$$

for $i=0,1, l+\frac{i m}{2} \leq m-1, k=2, \ldots, n-1$. Since

$$
\partial \bar{\partial} \rho\left(L_{n}^{\prime}, \bar{L}_{n}^{\prime}\right)=\frac{\partial^{2} \rho}{\partial \zeta_{n} \partial \bar{\zeta}_{n}}+\mathcal{\theta}(b),
$$

one gets by induction and (2.16) that

$$
\mathscr{L}_{j, k}^{\prime} \partial \bar{\partial} \rho=\frac{\partial^{j+k} \rho}{\partial \zeta_{n}^{j} \partial \bar{\zeta}_{n}^{k}}+E_{j+k-1}
$$


where

$$
\left|D_{k}^{i} D_{n}^{l} E_{s}(0)\right| \lesssim \delta \tau^{-l-s} \tau_{k}^{-i}, \quad l+\frac{i m}{2} \leq m-s,
$$

for $i=0,1,1 \leq s \leq m-1, k=2, \ldots, n-1$. With (2.17) and by induction one will get

$$
\left|D_{k}^{i} D_{n}^{l} \mathscr{L}_{j, k}^{\prime} \partial \bar{\partial} \rho(0)\right| \lesssim \delta \tau^{-(l+j+k)} \delta^{-\frac{i}{2}}
$$

and hence one obtains that

$$
\left|\mathscr{L}_{j, k} \partial \bar{\partial} r(z)\right| \lesssim \delta \tau^{-(j+k)}, \quad z \in Q_{\delta}\left(z^{\prime}\right),
$$

by a simple Taylor's theorem argument and (2.14). Since this means that $C_{l}(z) \lesssim \delta \tau^{-l}, z \in Q_{\delta}\left(z^{\prime}\right), l=2, \ldots, m$, one concludes that

$$
\eta(z, \delta) \gtrsim \tau\left(z^{\prime}, \delta\right),
$$

when $z \in Q_{\delta}\left(z^{\prime}\right)$. In the rest of this section we will show the opposite relation of (2.19). We first show that the quantities $B_{l}\left(z^{\prime}\right)$ in (2.6) are less important than $A_{l}\left(z^{\prime}\right)$ for the definition of $\tau\left(z^{\prime}, \delta\right)$ in (2.7). Recall that $L_{n}^{\prime}=\frac{\partial}{\partial \zeta_{n}}+b(\zeta) \frac{\partial}{\partial \zeta_{1}}$, where $b(\zeta)=-\left(\frac{\partial \rho}{\partial \zeta_{1}}\right)^{-1}\left(\frac{\partial \rho}{\partial \zeta_{n}}\right)$ satisfies (2.16). If one combines (2.4), (2.16), and Taylor's theorem, one will get

$$
|b(\zeta)| \lesssim \delta \tau^{-\frac{\xi}{4}}
$$

for $\zeta \in P_{\tau}=\left\{\zeta ;\left|\zeta_{1}\right| \leq \delta,\left|\zeta_{k}\right| \leq \delta^{\frac{1}{2}} \tau^{-\frac{1}{4}},\left|\zeta_{n}\right| \leq \tau\right\}$. Define a map $\Lambda_{\delta}: \mathbb{C}^{n} \longrightarrow$ $\mathrm{C}^{n}$ by

$$
\Lambda_{\delta}(\zeta)=\left(\delta^{-1} \zeta_{1}, \delta^{-\frac{1}{2}} \zeta_{2}, \ldots, \delta^{-\frac{1}{2}} \zeta_{n-1}, \tau^{-1} \zeta_{n}\right)=\left(\tilde{\zeta}_{1}, \ldots, \tilde{\zeta}_{n}\right)
$$

Then

$$
\tilde{L}_{n}=\tau\left(\Lambda_{\delta}\right)_{*} L_{n}^{\prime}=\frac{\partial}{\partial \zeta_{n}}+b\left(\Lambda_{\delta}^{-1}(\zeta)\right) \delta^{-1} \tau \frac{\partial}{\partial \zeta_{1}},
$$

where we have dropped the tildes in $\zeta$-variables. With (2.20), one has

$$
\left|b\left(\Lambda_{\delta}^{-1}(\zeta)\right) \delta^{-1} \tau\right| \lesssim \tau^{-\frac{1}{4}}
$$

for $\zeta \in Q_{\tau}=\Lambda_{\delta}\left(P_{\tau}\right)=\left\{\zeta ;\left|\zeta_{1}\right| \leq 1,\left|\zeta_{n}\right| \leq 1,\left|\zeta_{k}\right| \leq \tau^{-\frac{1}{\alpha}}, k=2, \ldots, n-1\right\}$. If we set $\rho_{z^{\prime}}^{\delta}(\zeta)=\delta^{-1}\left(\left(\Lambda_{\delta}^{-1}\right)^{*} \rho_{z^{\prime}}(\zeta)\right)$, then

$$
\begin{aligned}
\rho_{z^{\prime}}^{\delta}(\zeta)= & \operatorname{Re} \zeta_{1}+\sum_{\substack { \alpha=2 \\
\begin{subarray}{c}{j+k \leq \frac{m}{2} \\
j, k>0{ \alpha = 2 \\
\begin{subarray} { c } { j + k \leq \frac { m } { 2 } \\
j , k > 0 } }\end{subarray}} \operatorname{Re}\left[b_{j, k}^{\alpha}\left(z^{\prime}\right) \delta^{-\frac{1}{2}} \tau^{j+k} \zeta_{n}^{j} \bar{\zeta}_{n}^{k} \zeta_{\alpha}\right] \\
& +\sum_{\substack{j+k \leq m \\
j, k>0}} a_{j, k}\left(z^{\prime}\right) \delta^{-1} \tau^{j+k} \zeta_{n}^{j} \bar{\zeta}_{n}^{k}+\sum_{\alpha=2}^{n-1}\left|\zeta_{\alpha}\right|^{2} \\
& +\tau \theta\left(\left|\zeta_{1}\right||\zeta|+\delta\left|\zeta_{1}\right|^{2}|\zeta|+\left|\zeta^{\prime \prime}\right|^{2}|\zeta|+\left|\zeta^{\prime \prime}\right|\left|\zeta_{n}\right|^{\frac{m}{2}+1}+\left|\zeta_{n}\right|^{m+1}\right)
\end{aligned}
$$

for all $\zeta \in Q_{\tau}$. From the expression in (2.22), we set

$$
\begin{aligned}
& A^{\delta}\left(\zeta_{n}, \bar{\zeta}_{n}\right)=\sum_{\substack{j+k \leq m \\
j, k>0}} a_{j, k}\left(z^{\prime}\right) \delta^{-1} \tau^{j+k} \zeta_{n}^{j} \bar{\zeta}_{n}^{k}, \text { and } \\
& B_{\alpha}^{\delta}\left(\zeta_{n}, \bar{\zeta}_{n}\right)=\sum_{\substack{j+k \leq \frac{m}{2} \\
j, k>0}} b_{j, k}^{\alpha}\left(z^{\prime}\right) \delta^{-\frac{1}{2}} \tau^{j+k} \zeta_{n}^{j} \bar{\zeta}_{n}^{k}, \quad \alpha=2, \ldots, n-1 .
\end{aligned}
$$


Since the level sets of $\rho_{z^{\prime}}^{\delta}(\zeta)$ are pseudoconvex and since $\tilde{L}_{n}=\tau\left(\Lambda_{\delta}\right)_{*} L_{n}^{\prime}$ is a tangential vector field on the level sets of $\rho_{z^{\prime}}^{\delta}$, we have $\partial \bar{\partial} \rho_{z^{\prime}}^{\delta}(\zeta)\left(\tilde{L}, \overline{\tilde{L}}_{n}\right) \geq 0$. By combining (2.21) and (2.22), one can get

$$
\begin{aligned}
\partial \bar{\partial} \rho_{z^{\prime}}^{\delta}(\zeta)\left(\tilde{L}_{n}, \bar{L}_{n}\right) & =\frac{\partial^{2} \rho_{z^{\prime}}^{\delta}}{\partial \zeta_{n} \partial \bar{\zeta}_{n}}+\mathcal{\theta}\left(\tilde{b} \frac{\partial^{2} \rho_{z^{\prime}}^{\delta}}{\partial \zeta_{n} \partial \bar{\zeta}_{1}}\right)+\mathcal{\theta}\left(\tilde{b}^{2} \frac{\partial^{2} \rho_{z^{\prime}}^{\delta}}{\partial \zeta_{1} \partial \bar{\zeta}_{1}}\right) \\
& =\frac{\partial^{2} A^{\delta}}{\partial \zeta_{n} \partial \bar{\zeta}_{n}}+\operatorname{Re}\left(\sum_{\alpha=2}^{n-1} \frac{\partial^{2} B_{\alpha}^{\delta}\left(\zeta_{n}, \bar{\zeta}_{n}\right)}{\partial \zeta_{n} \partial \bar{\zeta}_{n}} \zeta_{\alpha}\right)+\mathcal{O}\left(\tau^{\frac{1}{2}}\right)
\end{aligned}
$$

for all $\zeta \in Q_{\tau}$ where $\tilde{b}=\delta^{-1} \tau b\left(\Lambda_{\delta}^{-1}(\zeta)\right)$.

Lemma 2.4. $\left|B_{\delta}^{\alpha}\left(\zeta_{n}, \bar{\zeta}_{n}\right)\right| \leq \tau$ to for all $\alpha=2, \ldots, n-1, \zeta \in Q_{\tau}$, provided $\tau$ is sufficiently small.

Proof. From (2.6) we know that the coefficients of $A^{\delta}$ and $B_{\alpha}^{\delta}$ are bounded by one. At first, let's show that $\left|\partial^{2} B_{\alpha}^{\delta}\left(\zeta_{n}, \bar{\zeta}_{n}\right) / \partial \zeta_{n} \partial \bar{\zeta}_{n}\right| \leq \tau^{\frac{1}{\text { to }}}$ for $\tau \in Q_{\tau}$. Suppose, on the contrary, that

$$
\left|\frac{\partial^{2} B_{\alpha}^{\delta}}{\partial \zeta_{n} \bar{\zeta}_{n}}\left(\zeta_{n}, \bar{\zeta}_{n}\right)\right|>\tau^{\frac{1}{10}}
$$

for some $\zeta_{n}$ and $\alpha$. Then

$$
\frac{\partial^{2} B_{\alpha}^{\delta}\left(\zeta_{n}, \bar{\zeta}_{n}\right)}{\partial \zeta_{n} \partial \bar{\zeta}_{n}} \zeta_{\alpha}<-\left|\theta\left(\tau^{-\frac{1}{10}}\right)\right|,
$$

provided one takes $\left|\zeta_{\alpha}\right|$ sufficiently large (say $\tau^{-\frac{1}{\zeta}}<\left|\zeta_{\alpha}\right|<\tau^{-\frac{1}{\alpha}}$ ), with appropriate argument. If one combines this fact and (2.23), then $\partial \bar{\partial} \rho_{z^{\prime}}^{\alpha}\left(\tilde{L}_{n}, \overline{\tilde{L}}_{n}\right)<0$ at that point provided $\tau$ is sufficiently small. Since the level sets of $\rho_{z^{\prime}}^{\delta}$ are pseudoconvex, this contradiction shows that $\left|\partial^{2} B_{\alpha}^{\delta}\left(\zeta_{n}, \bar{\zeta}_{n}\right) / \partial \zeta_{n} \partial \bar{\zeta}_{n}\right| \leq \tau \frac{1}{10}$. This implies that $\left|B_{\alpha}^{\delta}\left(\zeta_{n}, \bar{\zeta}_{n}\right)\right| \leq \tau$ 亩 because $\left|\zeta_{n}\right| \leq 1$.

Using this lemma, one can show that the coefficients of $B_{\delta}^{\alpha}$ can be made arbitrarily small provided $\delta$ is sufficiently small.

Lemma 2.5. Let $P_{k}(z, \bar{z})=\sum_{i+j=k} a_{i, j} z^{i} \bar{z}^{j}$ be a homogeneous polynomial of order $k$ in $z$ and $\bar{z}$, and suppose that $\left|P_{k}(z, \bar{z})\right| \leq \epsilon$ for all $z$ on the unit circle on $\mathbb{C}^{1}$. Then $\left|a_{i, j}\right| \leq \epsilon$.

Proof. $P_{k}(z, \bar{z})=\sum_{l+j=k} a_{l, j} e^{i(l-j) \theta}$ on the unit circle in $\mathbb{C}^{1}$. So

$$
\left|a_{l, j}\right|=\left|\frac{1}{2 \pi} \int_{-\pi}^{\pi} P_{k}(z, \bar{z}) e^{i(l-j) \theta} d \theta\right| \leq \frac{1}{2 \pi} \int_{-\pi}^{\pi}|P|_{\infty} d \theta \leq \epsilon .
$$

Proposition 2.6. Let $P(z, \bar{z})=\sum_{i+j \leq n} a_{i j} z^{i} \bar{z}^{j}$ be a polynomial of order $n$ with $\left|a_{i j}\right| \leq 1$. Suppose $|P(z, \bar{z})| \leq \epsilon^{2}$ for all $|z| \leq 1$ for some small number $\epsilon>0$. Then $\left|a_{i j}\right| \leq C_{n} \epsilon^{\alpha}$, where $\alpha=\frac{1}{n !}$.

Proof. Let $P=\sum_{k=0}^{n} P_{k}$, where $P_{k}$ is a homogeneous polynomial of order $k$. It is clear that $\left|P_{0}\right| \leq \epsilon^{2}$. Since $\left|\sum_{l=2}^{n} P_{l}\right| \lesssim \epsilon^{2}$ on $|z|=\epsilon$, we have $\left|P_{1}(z, \bar{z})\right| \leq$ 
$|P|+\left|P_{0}\right|+\left|\sum_{l=2}^{n} P_{l}\right| \lesssim \epsilon^{2}$ on $|z| \leq \epsilon$. This implies that $\left|P_{1}(z, \bar{z})\right| \leq \epsilon$ for all $|z| \leq 1$, and therefore $\left|a_{i j}\right| \leq \epsilon, i+j \leq 1$, by Lemma 2.5 . Similarly one can prove that $\left|P_{2}(z, \bar{z})\right| \leq \epsilon^{\frac{1}{2}}$ for all $|z| \leq 1$ and hence $\left|a_{i j}\right| \leq \epsilon^{\frac{1}{2}}, i+j \leq 2$. Let $k \geq 2$ and suppose by induction that $\left|a_{i j}\right| \lesssim \epsilon \frac{1}{k}$ for all $i+j \leq k$. Then $\left|\sum_{l=k+2}^{n} P_{l}\right| \lesssim \epsilon^{\frac{k+2}{k+1) !}}$ on $|z| \leq \epsilon^{\frac{1}{(k+1) !}}$, and so

$$
\left|\sum_{l=0}^{k} P_{l}\right| \lesssim\left|P_{0}\right|+\left|P_{1}\right|+\left|\sum_{l=2}^{k} P_{l}\right| \lesssim \epsilon^{\frac{1}{k !}+\frac{2}{(k+1) !}}
$$

on $|z| \leq \epsilon^{\frac{1}{(k+1) !}}$. Therefore

$$
\left|P_{k+1}\right| \lesssim|P|+\left|\sum_{l=k+2}^{n} P_{l}\right|+\left|\sum_{l=0}^{k} P_{l}\right| \lesssim \epsilon^{\frac{k+2}{k+1) !}}
$$

on $|z| \leq \epsilon^{\frac{1}{(k+1) !}}$. This implies that $\left|P_{k+1}\right| \lesssim \epsilon^{\frac{k+2}{(k+1) !}-\frac{k+1}{(k+1) !}}=\epsilon^{\frac{1}{(k+1) !}}$, for all $|z| \leq 1$, and hence $\left|a_{i j}\right| \leq \epsilon^{\frac{1}{(k+1) !}}$ for all $i+j \leq k+1$ by Lemma 2.5. So we get Proposition 2.6 by induction.

If one combines Lemma 2.4, Lemma 2.5 and Proposition 2.6, then

$$
\left|b_{j, k}^{\alpha}\left(z^{\prime}\right) \delta^{-\frac{1}{2}} \tau^{j+k}\right| \lesssim \tau \frac{1}{\text { खकxm! }}
$$

for all $2 \leq \alpha \leq n-1,2 \leq j+k \leq \frac{m}{2}$. So $\left(\delta^{\frac{1}{2}} / B_{l^{\prime}}\left(z^{\prime}\right)\right) \gg \tau, l^{\prime}=2, \ldots, \frac{m}{2}$, if $\delta$ (and hence $\tau$ ) is sufficiently small and therefore $\tau\left(z^{\prime}, \delta\right)=\min \left\{\left(\frac{\delta}{A_{1}\left(z^{\prime}\right)}\right)^{\dagger} ; 2 \leq\right.$ $l \leq m\}$. Now define

$$
T\left(z^{\prime}, \delta\right)=\min \left\{l ;\left(\delta / A_{l}\left(z^{\prime}\right)\right)^{\dagger}=\tau\left(z^{\prime}, \delta\right)\right\} .
$$

Then there exists $j, k$ with $j+k=T\left(z^{\prime}, \delta\right)$ so that

$$
\left|a_{j, k}\left(z^{\prime}\right)\right|=\left|\frac{\partial^{j+k} \rho}{\partial \zeta_{n}^{j} \partial \bar{\zeta}_{n}^{k}}(0)(j ! k !)^{-1}\right|=\delta \tau^{-j-k} .
$$

From (2.17), (2.18), and (2.26), one has

$$
\left|\mathscr{L}_{j, k}^{\prime} \partial \bar{\partial} \rho(0)\right| \approx(j ! k !) \delta \tau^{-j-k} .
$$

provided $\delta$ is sufficiently small. Again by Taylor's theorem argument and by the fact that $\left|\zeta_{n}\right|<\tau\left(z^{\prime}, b \delta\right) \leq b^{\frac{1}{m}} \tau\left(z^{\prime}, \delta\right)$ for $\zeta \in R_{b \delta}\left(z^{\prime}\right)$, one has

$$
\left|\mathscr{L}_{j, k}^{\prime} \partial \bar{\partial} \rho(\zeta)-\mathscr{L}_{j, k}^{\prime} \partial \bar{\partial} \rho(0)\right| \lesssim b^{\frac{1}{m}} \delta \tau^{-j-k},
$$

and hence $\left|\mathscr{L}_{j, k}^{\prime} \partial \bar{\partial} \rho(\zeta)\right| \approx \delta \tau^{-j-k}$ for $\zeta \in R_{b \delta}\left(z^{\prime}\right)$, if $b$ is sufficiently small. Therefore (2.27) together (2.13) give us $\eta(z, \delta) \lesssim \tau\left(z^{\prime}, \delta\right)$, for $\zeta \in Q_{b \delta}\left(z^{\prime}\right)$, and hence

$$
\eta(z, \delta) \lesssim b^{-\frac{1}{2}} \tau\left(z^{\prime}, \delta\right), \quad z \in Q_{\delta}\left(z^{\prime}\right),
$$

by (2.9). With (2.19) and (2.28), we have proved the following proposition. 
Proposition 2.7. Let $z^{\prime}$ and $z$ be any two points with $z \in Q_{\delta}\left(z^{\prime}\right)$. Then

$$
\tau\left(z^{\prime}, \delta\right) \lesssim \eta(z, \delta) \lesssim \tau\left(z^{\prime}, \delta\right) .
$$

Corollary 2.8. Suppose that $z \in Q_{\delta}\left(z^{\prime}\right)$. Then

$$
\tau\left(z^{\prime}, \delta\right) \approx \tau(z, \delta) \text {. }
$$

Proof. By Proposition 2.7, $\tau\left(z^{\prime}, \delta\right) \approx \eta(z, \delta) \approx \tau(z, \delta)$.

Using the definitions of $\eta\left(z^{\prime}, \delta\right), \tau\left(z^{\prime}, \delta\right), T\left(z^{\prime}, \delta\right)$ with Proposition 2.7 and Corollary 2.8, we can show the following semicontinuous result for the integer $T(z, \delta)$ by a method similar to Proposition 1.5 in [3].

Proposition 2.9. There exists a small constant $b>0$ so that if $z \in Q_{b \delta}\left(z^{\prime}\right)$, then

$$
T(z, \epsilon) \leq T\left(z^{\prime}, \delta\right)
$$

for all $\epsilon \leq b \delta$.

\section{Estimates OF the BERgMAN KERNEL FUNCTION}

In this section we prove the main theorem of this article. The following proposition is the local version of the problem constructing a function with large Hessian near the boundary. For $z$ near the boundary of $\Omega$, we denote the closest point in $b \Omega$ to $z$ by $\pi(z)$. Let us take the vector fields $L_{1}, \ldots, L_{n}$ as in (1.1).

Proposition 3.1. Suppose $z^{\prime} \in U \cap b \Omega$. Then there exist a small constant $a>0$ and a smooth function $g_{z^{\prime}, \delta}$ on $\bar{\Omega}$ that satisfies

(i) $\left|g_{z^{\prime}, \delta}(z)\right| \leq 1$ and $g_{z^{\prime}, \delta} \in C_{0}^{\infty}\left(Q_{\delta}\left(z^{\prime}\right)\right)$.

(ii) If $-a \delta \leq r(z) \leq a \delta$ and if $g_{z^{\prime}, \delta}$ is not plurisubharmonic at $z$, then

$$
T(\pi(z), a \delta)<T\left(z^{\prime}, \delta\right) .
$$

(iii) If $z \in Q_{a \delta}\left(z^{\prime}\right),-a \delta \leq r(z) \leq a \delta$, and if the inequality

$$
\partial \bar{\partial} g_{z^{\prime}, \delta}(L, \bar{L})(z) \gtrsim\left(\tau\left(z^{\prime}, \delta\right)\right)^{-2}\left|b_{n}\right|^{2}+\delta^{-1} \sum_{k=2}^{n-1}\left|b_{k}\right|^{2}+\delta^{-2}\left|b_{1}\right|^{2}
$$

fails to hold at $z$ for $L=\sum_{j=1}^{n} b_{j} L_{j}$, then

$$
T(\pi(z), a \delta)<T\left(z^{\prime}, \delta\right) \text {. }
$$

(iv) For all $z \in Q_{\delta}\left(z^{\prime}\right)$ and all $L=\sum_{j=1}^{n} b_{j} L_{j}$ at $z$,

$$
\left|\partial \bar{\partial} g_{z^{\prime}, \delta}(L, \bar{L})\right| \lesssim\left(\tau\left(z^{\prime}, \delta\right)\right)^{-2}\left|b_{n}\right|^{2}+\delta^{-1} \sum_{k=2}^{n-1}\left|b_{k}\right|^{2}+\delta^{-2}\left|b_{1}\right|^{2}
$$

(v) If $\Phi^{\prime}$ denotes the map associated with $z^{\prime}$, then

$$
\left|D^{\alpha} g_{z^{\prime}, \delta} \circ \Phi^{\prime}(\zeta)\right| \leq C_{\alpha} \tau^{-\alpha_{n}} \delta^{-\alpha_{1}} \delta^{-\frac{1}{2}\left(\alpha_{2}+\ldots+\alpha_{n-1}\right)}
$$

where $\alpha_{i}=\beta_{i}+\gamma_{i}, D^{\alpha}=D_{1}^{\alpha_{1}} \ldots D_{n}^{\alpha_{n}}$, and $D_{i}^{\alpha_{i}}=D_{i}^{\beta_{i}} \bar{D}_{i}^{y_{i}}$. 
Proof. The proof will be similar to that of dimension two case of Proposition 2.1 in [3]. We will sketch the proof briefly here. Set $\tau\left(z^{\prime}, \delta\right)=\tau$ for convenience. From (2.15), Proposition 2.3, and Lemma 2.4, one has

$$
\begin{aligned}
& \partial \bar{\partial} r\left(L_{k}, \bar{L}_{n}\right)=\partial \bar{\partial} \rho\left(L_{k}^{\prime}, \bar{L}_{n}^{\prime}\right)=\mathcal{O}\left(\delta^{\frac{1}{2}} \tau^{-1}\right), \text { and } \\
& \partial \bar{\partial} r\left(L_{k}, \bar{L}_{k}\right)=\partial \bar{\partial} \rho\left(L_{k}^{\prime}, \bar{L}_{k}^{\prime}\right)=1+\mathcal{O}\left(\delta^{\frac{1}{2}}\right),
\end{aligned}
$$

for $k=2, \ldots, n-1$. Therefore from Proposition 2.1 and the fact that $L r=$ $b_{1} L_{1} r$, we obtain that if $\delta$ is small and $\lambda \geq 1$,

$$
\begin{aligned}
\lambda \delta^{-1} \partial \bar{\partial} r(L, \bar{L})+\left(\lambda \delta^{-1}\right)^{2}|L r|^{2} \\
=\lambda \delta^{-1} \partial \bar{\partial} r\left(L_{n}, \bar{L}_{n}\right)\left|b_{n}\right|^{2}+2 \lambda \delta^{-1} \operatorname{Re} \sum_{j=1}^{n} \partial \bar{\partial} r\left(L_{1}, \bar{L}_{j}\right) b_{1} \bar{b}_{j} \\
\quad+\theta(\epsilon) \lambda \delta^{-1} \sum_{2 \leq j<k \leq n} b_{j} \bar{b}_{k}+\lambda \delta^{-1} \sum_{k=2}^{n-1} \partial \bar{\partial} r\left(L_{k}, \bar{L}_{k}\right)\left|b_{k}\right|^{2}+\lambda^{2} \delta^{-2}\left|b_{1} L_{1} r\right|^{2} \\
\approx \lambda \delta^{-1} \partial \bar{\partial} r\left(L_{n}, \bar{L}_{n}\right)\left|b_{n}\right|^{2}+\lambda \delta^{-1} \sum_{k=2}^{n-1}\left|b_{k}\right|^{2}+\lambda^{2} \delta^{-2}\left|b_{1}\right|^{2}+\mathcal{O}\left(\left|b_{n}\right|^{2}\right)
\end{aligned}
$$

Let $\psi(\zeta)$ be defined by

$$
\psi(\zeta)=\chi\left(\delta^{-2}\left|\zeta_{1}\right|^{2}+\delta^{-1} \sum_{k=2}^{n-1}\left|\zeta_{k}\right|^{2}+\tau^{-2}\left|\zeta_{n}\right|^{2}\right),
$$

where $\chi(t)=1$ for $t<\frac{b}{2}$ and $\chi(t)=0$ for $t \geq b$. Notice that $\psi(\zeta) \equiv 1$ if $\zeta \in Q_{b \delta}\left(z^{\prime}\right)$ for sufficiently small $b$. Here $b>0$ is the small constant as in Proposition 2.9. Now set $\Psi(z)=\psi\left(\left(\Phi_{z^{\prime}}\right)^{-1}(z)\right)$. Then by Proposition 2.3 and (2.15), one has

$$
\begin{gathered}
|\partial \bar{\partial} \Psi(L, \bar{L})|=\left|\partial \bar{\partial} \psi\left(L^{\prime}, \bar{L}^{\prime}\right)\right| \lesssim\left|b_{1}\right|^{2} \delta^{-2}+\delta^{-1} \sum_{k=2}^{n-1}\left|b_{k}\right|^{2}+\tau^{-2}\left|b_{n}\right|^{2}, \\
|L \Psi|=\left|L^{\prime} \psi\right| \lesssim\left|b_{1}\right| \delta^{-1}+\delta^{-\frac{1}{2}} \sum_{k=2}^{n-1}\left|b_{k}\right|+\tau^{-1}\left|b_{n}\right| .
\end{gathered}
$$

Suppose at first that $T\left(z^{\prime}, \delta\right)=2$. Then we conclude from (2.25) that

$$
\partial \bar{\partial} r\left(L_{n}, \bar{L}_{n}\right)(z) \approx \delta \tau^{-2}, \quad z \in Q_{b \delta}\left(z^{\prime}\right) .
$$

For $\lambda \geq 1$ we have

$$
\begin{aligned}
& \partial \bar{\partial}\left(\Psi e^{\lambda \delta^{-1} r}\right)(L, \bar{L})=e^{\lambda \delta^{-1} r}\left[\partial \bar{\partial} \Psi(L, \bar{L})+\lambda \delta^{-1} \sum_{i, j=1}^{n} 2 \operatorname{Re}\left(\left(L_{i} \Psi\right)\left(\bar{L}_{j} r\right)\right) b_{i} \bar{b}_{j}\right. \\
& \left.+\lambda \delta^{-1} \Psi \partial \bar{\partial} r(L, \bar{L})+\left(\lambda \delta^{-1}\right)^{2} \Psi|L r|^{2}\right] \text {. }
\end{aligned}
$$


Combining (3.5)-(3.8), one will get

$$
\partial \bar{\partial}\left(\Psi e^{\lambda \delta^{-1} r}\right)(L, \bar{L}) \approx \lambda\left(\delta^{-2}\left|b_{1}\right|^{2}+\delta^{-1} \sum_{k=2}^{n-1}\left|b_{k}\right|^{2}+\tau^{-2}\left|b_{n}\right|^{2}\right)
$$

provided $\lambda$ is sufficiently large and $\Psi(z) \geq \frac{1}{4}$.

Let $h$ denote a convex increasing smooth function such that $h(t)=0$ for $t \leq \frac{1}{2}$ and $h(t)>0$ for $t>\frac{1}{2}$, and set $g_{z^{\prime}}, \delta(z)=h\left(\Psi(z) e^{\lambda \delta^{-1} r(z)}\right)$. If $-a \delta \leq$ $r(z) \leq a \delta$, one has $\frac{3}{4}<e^{\lambda \delta^{-1} r(z)}<\frac{5}{4}$ provided $a>0$ is sufficiently small. This implies that $\Psi(z) \geq \frac{1}{4}$ if $z \in$ supp $g_{z^{\prime}, \delta}$ and $-a \delta \leq r(z) \leq a \delta$. Therefore $g_{z^{\prime}, \delta}(z)$ is smooth plurisubharmonic with support in $Q_{\delta}\left(z^{\prime}\right)$. It also satisfies property $(\mathrm{v})$ in Proposition 3.1 and hence this proves for the case of $T\left(z^{\prime}, \delta\right)=$ 2.

When $T\left(z^{\prime}, \delta\right)=l>2$, one has $\left|\mathscr{L}_{j, k} \partial \bar{\partial} r\left(z^{\prime}\right)\right| \approx \delta \tau^{-l}$ for some positive integers $j, k$ with $j+k=l$. This implies that at least one of the inequalities

$$
\left|L_{n}\left(\operatorname{Re} \mathscr{L}_{j-1, k} \partial \bar{\partial} r\right)\left(z^{\prime}\right)\right| \approx \delta \tau^{-l}
$$

and

$$
\left|L_{n}\left(\operatorname{Im} \mathscr{L}_{j-1, k} \partial \bar{\partial} r\right)\left(z^{\prime}\right)\right| \approx \delta \tau^{-l}
$$

is valid. (When $j=1$, we replace $\mathscr{L}_{j-1, k}$ by $\mathscr{L}_{1, k-1}$.) We may assume that (3.10) is valid. Now set $G(z)=\operatorname{Re} \mathscr{L}_{j-1, k} \partial \bar{\partial} r(z)$ and suppose that $T(z, e \delta)=$ $l$, for $e$ still to be chosen. Then by (2.6), (2.7), (2.12), (2.13) with Proposition 2.7 and Corollary 2.8 , one has

$$
\left|\mathscr{L}_{j-1, k} \partial \bar{\partial} r(z)\right| \leq C_{l-1}(z) \lesssim e^{t} \delta \tau^{-l+1},
$$

and by (2.18), one also has $\left|\partial \bar{\partial} G\left(L_{n}, \bar{L}_{n}\right)\right| \leq \delta \tau^{-l-1}$. Since $\partial \bar{\partial} G^{2}(L, \bar{L})(z)=$ $2|L G(z)|^{2}+2 G(z) \partial \bar{\partial} G(L, \bar{L})(z),(3.12)$ implies that

$$
\partial \bar{\partial} G^{2}\left(L_{n}, \bar{L}_{n}\right)(z) \geq c^{\prime} \delta^{2} \tau^{-2 l},
$$

provided $e>0$ is sufficiently small. Also from (2.15), (2.18), and Proposition 2.3, one has $\left|L_{k} G(z)\right| \lesssim \delta^{\frac{1}{2}} \tau^{-l+1}$, for $l=2, \ldots, m, k=2, \ldots, n-1$. The inequality $\left|\partial \bar{\partial} G^{2}\left(L_{i}, \widetilde{L}_{j}\right)\right| \lesssim 1$ is trivial for $i, j=1, \ldots, n$. Therefore we get

$$
\partial \bar{\partial} G^{2}(L, \bar{L})(z) \geq \frac{c^{\prime}}{2} \delta^{2} \tau^{-2 l}\left|b_{n}\right|^{2}-C^{\prime} \sum_{k=2}^{n-1} \delta \tau^{-2 l+2}\left|b_{k}\right|^{2}-C^{\prime}\left|b_{1}\right|^{2}
$$

Set

$$
G_{z^{\prime}, \delta}(z)=\Psi(z) e^{\lambda \delta^{-1} r(z)}+\phi\left(\delta^{-2} \tau^{2 l-2} G(z)^{2}\right)
$$

and set $g_{z^{\prime}, \delta}(z)=h\left(G_{z^{\prime}, \delta}(z)\right)$, where $\phi(t)$ is a smooth function that satisfies $\phi(t)=t, t \leq \frac{1}{16}, \phi(t)=0$ for $t \geq 1$, and $\phi(t) \leq \frac{1}{8}$ for all $t$. If one combines (3.5), (3.6), (3.8), (3.13), and the fact that $\delta^{-2} \tau^{2 l-2} G(z)^{2}<\frac{1}{16}$, provided $e$ is sufficiently small, one will get (3.2) and (3.3) and hence $g_{z^{\prime}, \delta}$ is plurisubharmonic for those $z \in Q_{b \delta}\left(z^{\prime}\right)$ with $T(z, e \delta)=l$. Now take $e \leq b$. Then Proposition 2.9 implies that

$$
T\left(\pi(z), e^{2} \delta\right) \leq T(z, e \delta) \leq T\left(z^{\prime}, \delta\right)=l
$$

for $z \in Q_{a \delta}\left(z^{\prime}\right)$, and $|r(z)| \leq a \delta$, where $a \leq e^{2}$. Therefore if $T(z, e \delta)<l$ then $T(\pi(z), a \delta)<l$ and hence this proves (ii)-(iv). For (i), we divide $g_{z^{\prime}, \delta}$ 
by some constant. Since $g_{z^{\prime}, \delta}(z)$ is a composition of the functions which satisfy (3.4), it also satisfies (3.4) and this proves (v).

Using Proposition 3.1, we can prove the following proposition which says that there is a bounded plurisubharmonic weight function such that the Hessian satisfies certain essentially maximal bounds in a thin strip near the boundary of $\Omega$. For $\epsilon>0$, we let $\Omega_{\epsilon}=\{z ; r(z)<\epsilon\}$ and set $S(\epsilon)=\{z:-\epsilon<r(z)<\epsilon\}$.

Theorem 3.2. For all small $\delta>0$, there is a plurisubharmonic function $\lambda_{\delta} \in$ $C^{\infty}\left(\Omega_{\delta}\right)$ with the following properties:

(i) $\left|\lambda_{\delta}(z)\right| \leq 1, z \in U \cap \Omega_{\delta}$.

(ii) For all $L=\sum_{j=1}^{n} b_{j} L_{j}$ at $z \in U \cap S(\delta)$,

$$
\partial \bar{\partial} \lambda_{\delta}(z)(L, \bar{L}) \approx \delta^{-2}\left|b_{1}\right|^{2}+\delta^{-1} \sum_{k=2}^{n-1}\left|b_{k}\right|^{2}+\tau^{-2}\left|b_{n}\right|^{2},
$$

(iii) If $\Phi_{z^{\prime}}$ is the map associated with a given $z^{\prime} \in U \cap S(\delta)$, then for all $\zeta \in R_{\delta}\left(z^{\prime}\right)$ with $|\rho(\zeta)|<\delta$,

$$
\left|D^{\alpha}\left(\lambda_{\delta} \circ \Phi_{z^{\prime}}\right)(\zeta)\right| \lesssim C_{\alpha} \delta^{-\alpha_{1}} \delta^{-\frac{1}{2}\left(\alpha_{2}+\cdots+\alpha_{n-1}\right)} \tau^{-\alpha_{n}}
$$

where $\alpha=\left(\alpha_{1}, \ldots, \alpha_{n}\right)$.

Proof. In the proof of Theorem 3.1 in [3], Catlin used only the properties of functions in Proposition 3.1 here. The proof of Theorem 3.2 is therefore essentially the same as the proof of Theorem 3.1 in [3], so we omit it.

The following theorem was essentially done by Hörmander and Catlin has modified it in [3].

Theorem 3.3. Let $\Omega$ be a bounded pseudoconvex domain in $\mathbb{C}^{n}$ with smooth boundary. Assume that $z^{\prime}=\left(z_{1}^{\prime}, \ldots, z_{n}^{\prime}\right)$ is a given point in $\Omega$, that $\tau_{1}, \ldots$, $\tau_{n}$ are given positive numbers, and that there is a function $\phi \in C^{3}(\bar{\Omega})$ that satisfies the following properties:

(i) $|\phi(z)| \lesssim 1, z \in \Omega$.

(ii) $\phi$ is plurisubharmonic in $\Omega$.

(iii) $\Omega$ contains the polydisc $B=\left\{z ;\left|z_{i}-z_{i}^{\prime}\right|<\tau_{i}, i=1, \ldots, n\right\}$.

(iv) In $\Omega, \phi$ satisfies

$$
\sum_{i, j=1}^{n} \frac{\partial^{2} \phi}{\partial z_{i} \partial \bar{z}_{j}}(z) t_{i} \bar{t}_{j} \gtrsim \sum_{i=1}^{n} \tau_{i}^{-2}\left|t_{i}\right|^{2}, \quad z \in B .
$$

(v) If $D_{i}^{\alpha_{i}}$ denotes any mixed partial derivatives in $z_{i}$ and $\bar{z}_{i}$ of total order $\alpha_{i}$, then $D^{\alpha} \phi=D_{1}^{\alpha_{1}} \ldots D_{n}^{\alpha_{n}} \phi$ satisfies

$$
\left|D^{\alpha} \phi(z)\right| \lesssim C_{\alpha} \prod_{i=1}^{n} \tau_{i}^{-\alpha_{i}}, \quad z \in B, \quad|\alpha| \leq 3 .
$$


Then $K_{\Omega}\left(z^{\prime}, \bar{z}^{\prime}\right)$, the Bergman kernel function of $\Omega$ at $z^{\prime}$, satisfies

$$
K_{\Omega}\left(z^{\prime}, \bar{z}^{\prime}\right) \approx \prod_{i=1}^{n} \tau_{i}^{-2}
$$

We now ready to prove Theorem 1. Let $z \in U$ with $r(z)=-\frac{b \delta}{2}$ and $\pi(z)=z^{\prime} \in b \Omega$ where $U$ is a small neighborhood of $z_{0} \in b \Omega$ and $b>0$ is the number as in Proposition 2.9. Set $\phi_{\delta}^{\prime}(\zeta)=\lambda_{\delta} \circ \Phi_{z^{\prime}}(\zeta)$. Here $\Phi_{z^{\prime}}$ is the map in Proposition 2.2. Then $\phi_{\delta}^{\prime}$ will satisfy Theorem 3.3 in $\zeta$-coordinates. So we will work on $\Omega_{z^{\prime}}=\left(\Phi_{z^{\prime}}^{-1}\right)(\Omega)$. Set $\zeta=\left(-\frac{b \delta}{2}, 0, \ldots, 0\right)$. Then $\zeta=\Phi_{z^{\prime}}^{-1}(z)$ and by (2.11) there is a constant $0<c<1$ such that the polydisc $B=\{\zeta$ : $\left.\left|\zeta_{1}+b \delta / 2\right|<c \delta, \quad\left|\zeta_{k}\right|<c \delta^{\frac{1}{2}}, \quad\left|\zeta_{n}\right|<c \tau\left(z^{\prime}, \delta\right), k=2, \ldots, n-1\right\}$ lies in $\Omega_{z^{\prime}}$ and $\phi_{\delta}^{\prime}$ satisfies (3.14) on $B$. Hence

$$
K_{\Omega_{z^{\prime}}}(\zeta, \bar{\zeta}) \approx \delta^{-2} \delta^{-(n-2)} \tau\left(z^{\prime}, \delta\right)^{-2}=\delta^{-n} \tau\left(z^{\prime}, \delta\right)^{-2}
$$

by (3.15). Since the Jacobian of $\Phi_{z^{\prime}}$ at $\zeta$ satisfies

$$
\left|J_{\zeta}\left(\Phi_{z^{\prime}}\right)\right|=\left|\operatorname{det}\left[\frac{\partial \Phi_{z^{\prime}}^{i}}{\partial \zeta_{j}}(\zeta)\right]\right| \approx 1,
$$

the transformation identity of the Bergman kernel function implies that

$$
K_{\Omega}(z, \bar{z})=\left|J_{\zeta}\left(\Phi_{z^{\prime}}\right)\right|^{-2} K_{\Omega_{z^{\prime}}}(\zeta, \bar{\zeta}) \approx \delta^{-n} \tau\left(z^{\prime}, \delta\right)^{-2} .
$$

Since $z \in Q_{\delta}\left(z^{\prime}\right)$, we have $\tau\left(z^{\prime}, \delta\right) \approx \eta(z, \delta)$ by $(2.29)$. So from the definition of $\eta(z, \delta)$ in $(2.13)$ and from the fact that $|r(z)| \approx \delta$, we have

$$
\eta(z, \delta)^{-2} \approx \sum_{l=2}^{m}\left|C_{l}(z)\right|^{3}|r(z)|^{-3}
$$

and hence

$$
K_{\Omega}(z, \bar{z}) \approx|r(z)|^{-n}(\eta(z, \delta))^{-2} \approx \sum_{l=2}^{m}\left|C_{l}(z)\right|^{2}|r(z)|^{-n-z}
$$

The proof of Theorem 1 is now complete.

Remark 3.1. Theorem 3.2 says that the optimal subelliptic estimates (of order $m$ ) hold near $z_{0}$ according to the Catlin's theorem in [2]. Also the functions constructed in Theorem 3.2 will be useful for other purposes. These include unform extendability in a pseudoconvex way of maximal order $m$ and the estimates of Bergman kernel function off the diagonal.

Remark 3.2. The optimal estimates for the Caratheodory, Kobayashi, and Berg man metrics will be obtained in a forthcoming article using the theorems in this article and [4].

\section{REFERENCES}

1. E. Bedford and J. E. Fornaess, Biholomorphic maps of weakly pseudoconvex domains, Duke Math. J. 45 (1978), 711-719.

2. D. W. Catlin, Subelliptic estimates for the $\bar{\partial}$-Neumann problem on pseudoconvex domains, Ann. of Math. (2) 126 (1987), 131-191. 
3. 200 (1989), 429-466.

4. S. Cho, Extension of complex structures on weakly pseudoconvex compact complex manifolds with boundary, Math. Z. 211 (1992), 105-120.

5. __, A lower bound on the Kobayashi metric near a point of finite type in $\mathrm{C}^{n}, \mathrm{~J}$. Geom. Anal. 2 (1992), 317-325.

6. J. D'Angelo, A note on the Bergman kernel, Duke Math. J. 45 (1978), 259-266.

7. _- Real hypersurfaces, order of contact, and applications, Ann. of Math. (2) 115 (1982), 615-637.

8. K. Diederich and J. Fornaess, Proper holomorphic maps onto pseudoconvex domains with real-analytic boundary, Ann. of Math. (2) 110 (1979), 575-592.

9. K. Diederich, J. Fornaess and G. Herbort, Boundary behavior of the Bergman metric, Proc. Sympos. Pure Math., vol. 41, Amer. Math. Soc., Providence, RI, 1982, pp. 59-67.

10. K. Diederich, G. Herbort T. and Ohsawa, The Bergman kernel on uniformly extendable Pseudoconvex domains, Math. Ann. 237 (1986), 471-478.

11. Ch. Fefferman, The Bergman kernel and biholomorphic mappings of pseudoconvex domains, Invent. Math. 26 (1974), 1-65.

12. G. Herbort, The growth of the Bergman kernel on pseudoconvex domains of homogeneous finite diagonal type, Nagoya Math. J. 126 (1992), 1-24.

13. L. Hörmander, An introduction to complex analysis in several variables, North-Holland, Amsterdam, 1979.

14. J. J. Kohn, Boundary behavior of $\bar{\partial}$ on weakly pseudoconvex manifolds of dimension two, J. Difierential Geom. 6 (1972), 523-542.

15. J. McNeal, Lower bounds on the Bergman metric near a point of finite type, Ann. of Math. (2) 136 (1992), 339-360.

16. T. Ohsawa, Boundary behavior of the Bergman kernel function on pseudoconvex domains, Publ. Res. Inst. Math. Sci. Kyoto Univ. 20 (1984), 897-902.

Department of Mathematics Education, Pusan University, Pusan 609-735, Korea

E-mail address: choehyonon.pusan.ac.kr 